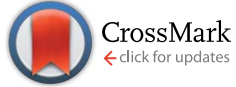

Cite this: RSC Adv., 2016, 6, 93881

Received 25th August 2016

Accepted 26th September 2016

DOI: 10.1039/c6ra21399e

www.rsc.org/advances

\section{Direct reductive coupling of indoles to nitrostyrenes en route to (indol-3-yl)acetamides $\uparrow$}

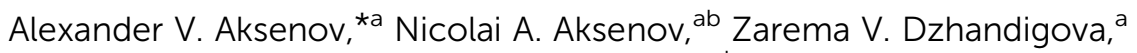 \\ Dmitrii A. Aksenov, ${ }^{a}$ Leonid G. Voskressensky, ${ }^{b}$ Valentine G. Nenajdenko ${ }^{c}$ \\ and Michael Rubin*ad
}

A highly efficient one-pot method for the reductive coupling of indoles to nitrostyrenes in polyphosphoric acid doped with $\mathrm{PCl}_{3}$ was developed. This method allows direct and expeditious access to primary (indol-3yl)acetamides, interesting as anti-cancer drug candidates.

\section{Introduction}

The (indol-3-yl)acetamide unit is omnipresent in biologically active compounds of both natural and synthetic origins. For example, this moiety was found in the structure of several argiotoxins, natural glutamate receptor antagonists isolated from the venom of an orb weaving spider Agriope aurantia. ${ }^{1}$ This fragment also serves as a core in the structures of many medicinal agents. Among these are microtubule inhibitor Indibulin, ${ }^{2}$ selective ATP-competitive p38 inhibitor Talmapimod, ${ }^{3}$ and commercial non-steroidal anti-inflammatory drug Indometacin ${ }^{4}$ (Fig. 1), to name a few. Recently we have reported on the promising anti-tumor activity of 2-aryl-2-(3-indolyl) acetohydroxamic acids 4 isolated as stable intermediates in unusual ANRORC reaction of indoles 1 with nitrostyrenes 2 affording 2-quinolones 5 (Scheme 1). ${ }^{5}$ Hydroxamic acids 4 resulting from polyphosphoric acid-assisted isomerization of nitroalkanes 3 demonstrated significant activity against melanoma, glioma, esophageal, and many other cancer lines intrinsically resistant to apoptosis induction and poorly responsive to treatment with traditional proapoptotic drugs. ${ }^{6}$ It was also shown that related nitriles 6 and amides 7 have also demonstrated decent levels of anti-cancer activity. ${ }^{6}$ Methods for efficient preparation of nitriles 6 either by reduction of hydroxamic acids 4 (ref. 7) or by direct reductive coupling of starting materials 1 and $2,{ }^{8}$ were developed in our laboratories

${ }^{a}$ Department of Chemistry, North Caucasus Federal University, 1a Pushkin St., Stavropol 355009, Russian Federation. E-mail: alexaks05@rambler.ru

${ }^{b}$ Department of Organic Chemistry, People's Friendship University of Russia, 6 Miklukho-Maklaya St., Moscow, 117198, Russian Federation

'Department of Chemistry, Lomonosov Moscow State University, Moscow 119899, Russia. E-mail: nenajdenko@org.chem.msu.ru

${ }^{d}$ Department of Chemistry, University of Kansas, 1251 Wescoe Hall Dr., Lawrence, KS 66045-7582, USA. E-mail: mrubin@ku.edu; Fax: +1-785-864-5396; Tel: +1-785-8645071

$\dagger$ Electronic supplementary information (ESI) available: Experimental procedures, physico-chemical and spectral data. See DOI: 10.1039/c6ra21399e
(Scheme 1). Acetamides 7, however, were only available in moderate yields via an acid-assisted hydrolysis of nitriles 6 (Scheme 1). ${ }^{6}$ This multi-step approach proved impractical for rapid assembly of the amide series that we needed for our ongoing SAR studies. We were in search for an efficient and expeditious one-pot method for direct assembly of such amides. Herein we wish to report on our progress in this investigation.

\section{Results and discussion}

Polyphosphoric acid (PPA) is often employed in our laboratories as an ionic liquid-like reaction medium for carrying out cascade and one-pot transformations involving acid-catalyzed steps,<smiles>N=C(N)NCCC[C@H](N)C(=O)NCCCCCNC(=O)[C@H](CC(N)=O)NC(=O)Cc1c[nH]c2cccc(O)c12</smiles><smiles>O=C(Nc1ccncc1)C(=O)c1cn(Cc2ccc(Cl)cc2)c2ccccc12</smiles><smiles>COc1ccc2c(c1)c(CC(N)=O)c(N)n2C(=O)c1ccc(Cl)cc1</smiles><smiles>CC1CN(C(=O)c2cc3c(CC(=O)N(C)C)cn(C)c3cc2Cl)C(C)CN1Cc1ccc(F)cc1</smiles>

Fig. 1 Important biologically active 3-indolylacetamides. 
<smiles>[R]c1cc2ccccc2n1[R]</smiles>

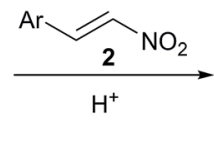<smiles>[R]c1c(C(Br)C[N+](=O)[O-])c2ccccc2n1[R]</smiles>

$[6]$ PPA, $70^{\circ} \mathrm{C}$

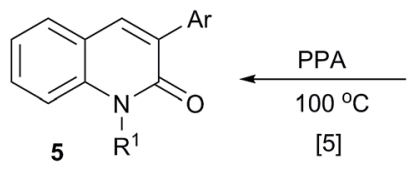<smiles>[R]c1c(C([Al])C(=O)NO)c2ccccc2n1[Z1]</smiles>

$\downarrow[7,8]$<smiles>[R]c1c(C([Al])C(N)=O)c2ccccc2n1[R]</smiles>
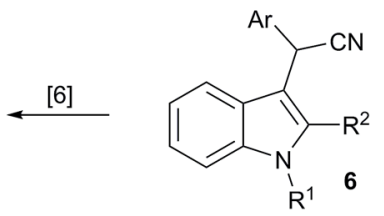

Scheme 1

such as electrophilic aromatic substitution, Schmidt and Beckmann rearrangements, Vilsmeier-type reactions, nucleophilic substitutions, as well variety of acid-promoted eliminations and condensations. Properties of this material strongly depend on concentration, thus in our labs, we normally obtain it by dissolving measured amounts of phosphorus pentoxide $\left(\mathrm{P}_{2} \mathrm{O}_{5}\right)$ in a known volume of hot orthophosphoric acid $\left(\mathrm{H}_{3} \mathrm{PO}_{4}\right)$. During our work on synthesis of hydroxamic acids 4 (ref. 6) we acquired a commercial sample of phosphorus pentoxide, containing impurities of red phosphorus left behind due to an incomplete combustion. Polyphosphoric acid, which was prepared from such $\mathrm{P}_{2} \mathrm{O}_{5}$, was also contaminated with finely dispersed red phosphorous and had a distinct pink color. Reaction between 2-phenylindole (1a) and $\beta$-nitrostyrene (2a) carried out in this "Pink PPA" afforded mixtures of hydroxamic acid 4 aa (about $40 \%$ ) and unexpected amide 7aa (also about 40\%) (Scheme 2). This serendipitous discovery prompted us to explore opportunities for a single

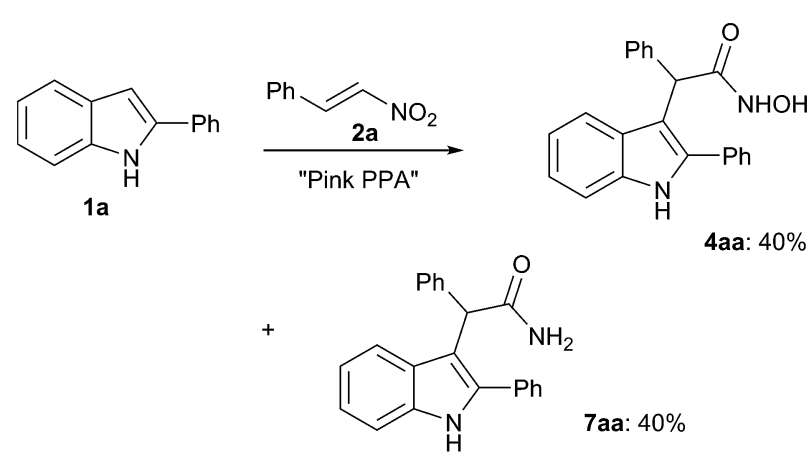

Scheme 2 step synthesis of amides 7 directly from indoles 1 and nitroolefins 2. It is known that hydroxamic acids can be converted to amides via catalytic hydrogenation, ${ }^{9}$ metal-catalyzed reductions and reductive coupling reactions, ${ }^{\mathbf{1 0}}$ in enzymatic processes, ${ }^{11}$ or in reaction with thiolphenols. ${ }^{12}$ We reasoned, that by doping PPA with an appropriate reducing agent we should be able to combine our synthesis of hydroxamic acids 4 with a reduction step (dashed red arrow in Scheme 1) in a sequential transformation to obtain pure amides 7. First, we attempted to reproduce preparation of "Pink PPA" by mixing pure $80 \%$ PPA with red phosphorus. This reagent, however, demonstrated much lower reducing power, probably, due to bigger particle sizes in commercially available phosphorus sample. In reaction between $1 \mathrm{a}$ and $2 \mathbf{a}$ carried out in this medium at $70{ }^{\circ} \mathrm{C}$ we managed to afford only $12 \%$ of amide $7 \mathbf{a a}$. An attempt to drive the reduction to completion by increasing the temperature to $100{ }^{\circ} \mathrm{C}$ did not lead to improved yield of amide, instead hydroxamic acid $4 \mathbf{a a}$ isomerized into corresponding quinolone $5 \mathbf{a a}\left(\mathrm{R}^{1}=\mathrm{H}, \mathrm{Ar}=\mathrm{Ph}\right)$. Next we tested other mild reducing agents, such as elementary sulfur and selenium, thiourea, triethyl- and trimethylphospites, or phosphorous acid (all these reagents were used in equimolar amounts). Unfortunately, these reactions did not afford any amide at all. Small quantities of amide 7aa (NMR yields are listed in parentheses) were detected in reactions carried out in PPA in the presence of $\mathrm{Ph}_{3} \mathrm{P}(10 \%), \mathrm{Et}_{2} \mathrm{~S}(4 \%)$, or $\mathrm{PCl}_{3}(32 \%)$. The last reagent was selected to be advanced to the next round of optimization, which included doubling the amount of the reducing agent. To this end, $0.25 \mathrm{M}$ solution of $1 \mathrm{a}$ and $2 \mathrm{a}$ in $80 \%$ PPA was stirred with 2 equiv. of $\mathrm{PCl}_{3}$ at $50-55{ }^{\circ} \mathrm{C}$ for $1 \mathrm{~h}$ to afford 7 aa in $54 \%$ yield. Similar experiment performed with more concentrated solution $(0.5 \mathrm{M})$ allowed to boost the yield up to $85 \%$ (Scheme 3 ), which we did not manage to improve any higher. With optimized conditions in hands employing variously substituted indoles $\mathbf{1 a - d}$ and nitrostyrenes $\mathbf{2 a - e}$ we managed to prepare a series of the requested amides in good to excellent yields (Scheme 3). Reaction with nitroethylene $\mathbf{2 f}$ was more challenging since this olefin tends to polymerize in the presence of acids. Nevertheless, $1 H$-indole-3-acetamides $7 \mathbf{b f}$ and 7 ef could also be accessed by the described method, albeit in moderate yields (Scheme 3 ).

We also were intrigued to test the possibility of employing this method for a direct and practical preparation of diarylacetamides 9 (Scheme 4). Indeed, we expected, that since the C-C bond-forming step involves nucleophilic conjugate addition to nitro-olefin 2 , electron rich arenes could also be used as nucleophiles in this reaction. We tested reactions between nitrostyrene (2a) and anisole (8a) under standard conditions, which provided 2-(4-methoxyphenyl)-2-phenylacetamide (9aa) in $71 \%$ yield (Scheme 4). Reactions of anisole (8a) with nitroethylene (2f) and 1-nitropropene (2g) were carried out under similar conditions to give the corresponding products 9af and 9ag (4 equiv. of nitroalkene should be loaded to compensate for partial losses due to polymerization). Similarly, reactions of $\mathbf{2 a}$ involving benzodioxane $\mathbf{8 b}$, and benzo-crown ethers (8c) also proceeded smoothly, affording the corresponding acetamides 9ba, 9ca albeit in moderate yields. 
<smiles>[R]c1cc2ccccc2[nH]1</smiles>
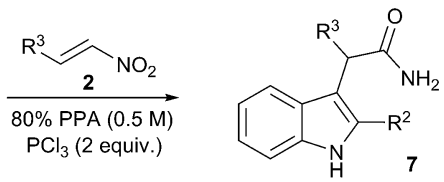

1a: $R^{2}=P h ; 1 \mathbf{b}: R^{2}=M e$

1c: $\mathrm{R}^{2}=4-\mathrm{MeC}_{6} \mathrm{H}_{4}$;

1d: $R^{2}=$ 2-naphthyl; 1e: $R^{2}=H$

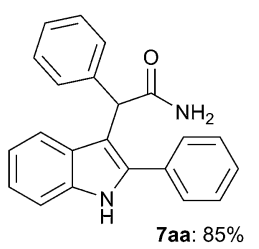

2a: $\mathrm{R}^{3}=\mathrm{Ph} ; \mathbf{2 b}: \mathrm{R}^{3}=4-\mathrm{EtC}_{6} \mathrm{H}_{4}$

2c: $\mathrm{R}^{3}=4-\mathrm{MeOC}_{6} \mathrm{H}_{4} ; 2 \mathrm{~d}: \mathrm{R}^{3}=2-\mathrm{FC}_{6} \mathrm{H}_{4}$

2e: $\mathrm{R}^{3}=4-\mathrm{ClC}_{6} \mathrm{H}_{4} ; \mathbf{2 f}: \mathrm{R}^{3}=\mathrm{H}$
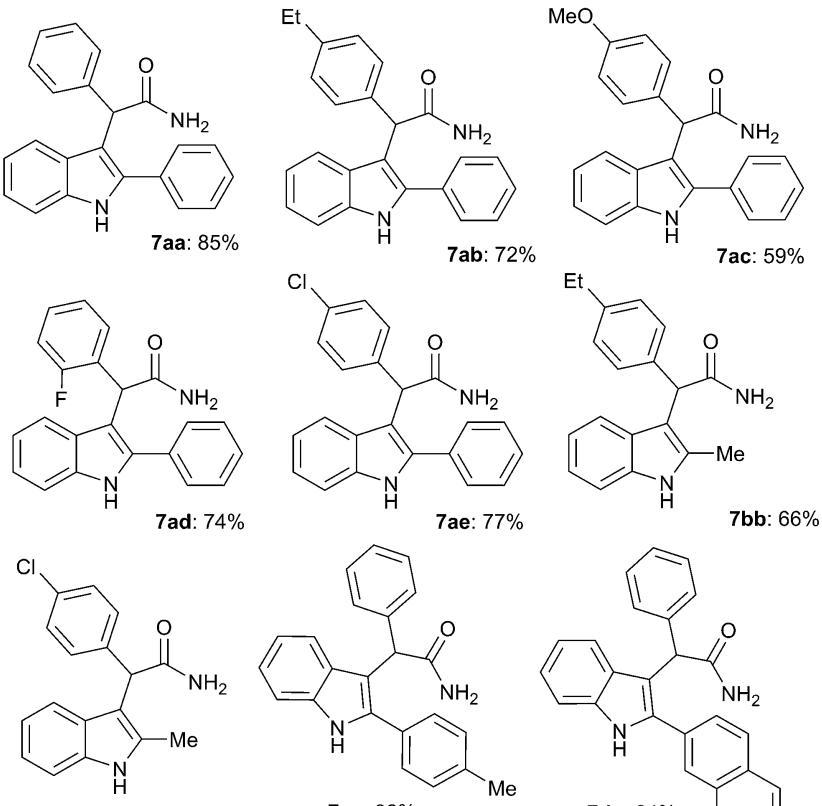<smiles>NC(=O)C(c1ccccc1)c1c(-c2ccc([N+](=O)[O-])cc2)[nH]c2ccccc12</smiles>

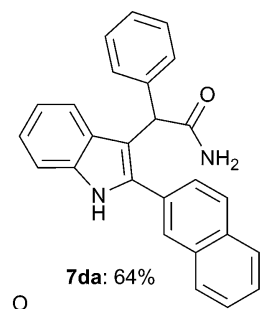

7ca: $82 \%$

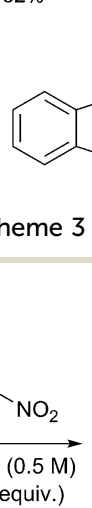<smiles>[X]c1ccccc1[X]</smiles>

8a: $X=H, Y=O M e$; 8b: $X Y=\mathrm{OCH}_{2} \mathrm{CH}_{2} \mathrm{O}$; 8c: $X Y=\mathrm{O}\left(\mathrm{CH}_{2} \mathrm{CH}_{2} \mathrm{O}\right)_{4}$;<smiles>COc1ccc(C(C(N)=O)c2ccccc2)cc1</smiles>

$$
\frac{2}{80 \% \operatorname{PPA}(0.5 \mathrm{M})}
$$<smiles>[X]c1ccc(C([R])C(N)=O)cc1[X]</smiles>

9 9aa: $71 \%$

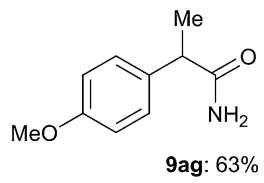<smiles>NC(=O)C(c1ccccc1)c1ccc2c(c1)OCCO2</smiles><smiles>COc1ccc(CC(N)=O)cc1</smiles>

$a: R^{3}=P h$

$\mathrm{R}^{3}=\mathrm{H}$<smiles>NC(=O)C(c1ccccc1)c1ccc2c(c1)OCCOCCOCCOCCO2</smiles>

Scheme 4
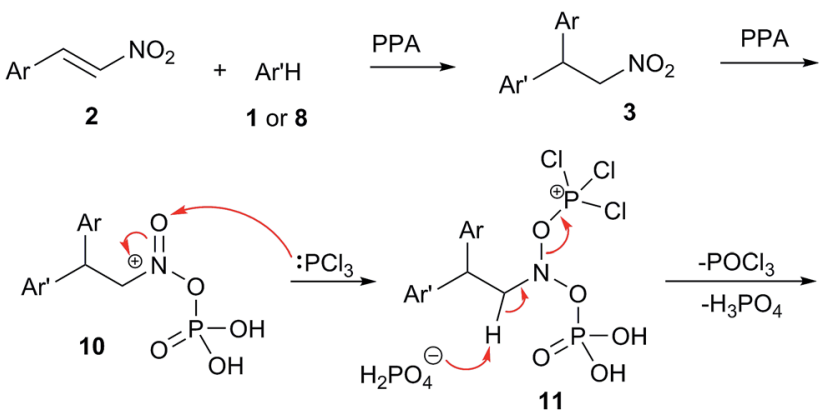<smiles>C[CH+]C</smiles>

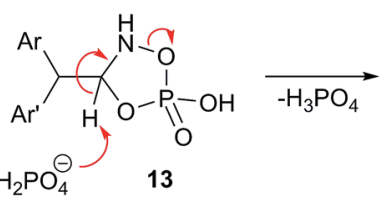<smiles>N=C(OP(=O)([O-])O)C([Al])[Al]</smiles>

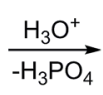<smiles>NC(=O)C([Al])Br</smiles>

Scheme 5

We believe that a plausible mechanism of the featured transformation may involve the following steps depicted in Scheme 5. Initial conjugate nucleophilic addition of indole $\mathbf{1}$ or electron-rich arene 8 to nitroalkene 2 would provide nitroalkane 3 (it should be pointed out, that authentic sample of molecule $3\left(\mathrm{Ar}=\mathrm{Ph}, \mathrm{Ar}^{\prime}=p\right.$ $\mathrm{MeOC}_{6} \mathrm{H}_{4}$ ) subjected to the reaction under the same conditions smoothly yielded expected product 9aa), which could be $O$-phosphorylated in the presence of PPA to afford species 10. The latter further undergoes nucleophilic attack by $\mathrm{PCl}_{3}$ to afford trichlorooxyphosphonium derivative 11, which after elimination of $\mathrm{POCl}_{3}$ would yield $O$-phosphorylated oxime 12. Subsequent intramolecular nucleophilic attack of phosphate moiety across $\mathrm{C}=\mathrm{N}$ bond of oxime shall provide dioxazaphospholidine species 13, further experiencing elimination of hydrophosponate entity leading to the formation of acetimidic phosphoric anhydride $\mathbf{1 4}$ (shown in deprotonated form). Finally, after aqueous work up ultimate product, acetamide 7 (or 9) would be afforded (Scheme 5). Remarkably, in the reaction operating via the suggested mechanism no should produce only inorganic byproducts. Indeed, we were pleased to notice that all the tested reactions proceeded cleanly affording target amide as sole isolable products along with variable quantities of readily separable polymeric resins.

\section{Conclusions}

We have developed an efficient cascade transformations involving formation of $\mathrm{C}-\mathrm{C}$ bond via conjugate nucleophilic addition of indoles (or electron-rich arenes) to 2-nitrostyrenes followed by reductive transformation of $\mathrm{CH}_{2} \mathrm{NO}_{2}$ group into carboxamide function. These processes allows for a single-step assembly of (indol-3-yl)acetamides and diarylacetamides in practical yields. Application of this method towards synthesis of libraries of amides 7 and SAR studies of their anti-tumor activity are currently under way in our laboratories. 


\section{Experimental part}

${ }^{1} \mathrm{H}$ and ${ }^{13} \mathrm{C}$ NMR spectra were recorded on a Bruker Avance-III spectrometer (400 or $100 \mathrm{MHz}$, respectively) equipped with BBO probe in DMSO- $d_{6}$, using TMS as internal standard. Highresolution mass spectra were registered with a Bruker Maxis spectrometer (electrospray ionization, in MeCN solution, using $\mathrm{HCO}_{2} \mathrm{Na}-\mathrm{HCO}_{2} \mathrm{H}$ for calibration). Melting points were measured with a Stuart smp30 apparatus. All reactions were performed in oven-dried $5 \mathrm{~mL}$ Erlenmeyer flasks open to the atmosphere, employing overhead stirring. Reaction progress and purity of isolated compounds were controlled by TLC on Silufol UV-254 plates. Reagents and solvents were purchased from commercial vendors and used as received.

\section{General procedure for preparation of amides}

Reaction vessel was charged with $80 \%$ PPA (1.2 g), phosphorus trichloride (137 mg, $1.0 \mathrm{mmol}$ ), and arene (or indole) (0.5 $\mathrm{mmol})$. The mixture was stirred at $50-55{ }^{\circ} \mathrm{C}$ for $5 \mathrm{~min}$ for homogenization, and nitrostyrene $(0.5 \mathrm{mmol})$ was added in a single portion. Nitroethylene (2f) and 1-nitropropene (2 g) were employed in excess $(2 \mathrm{mmol}, 4$ equiv.). Stirring of the mixture was continued for $3 \mathrm{~h}$, and the reaction progress was controlled by TLC. Then, the reaction mixture was poured out into stirred cold water $(20 \mathrm{~mL})$, and the mixture was basified with aqueous ammonia to $\mathrm{pH}$ 8. The product was extracted with ethyl acetate $(4 \times 20 \mathrm{~mL})$ and purified by Flash column chromatography on silica gel eluting with mixture of EtOAc and petroleum ether (from $1: 5$ to $1: 1$ ), or mixture of EtOH and EtOAc (1:10, used for purification of 9ca). Alternatively crude crystalline product could be re-crystallized from ethanol.

2-Phenyl-2-(2-phenyl-1H-indol-3-yl)acetamide (7aa). Colorless solid, mp 139-141 ${ }^{\circ} \mathrm{C}(\mathrm{EtOH}), R_{\mathrm{f}} 0.56$ (EtOAc/petroleum ether $1: 1)=0.56 ;{ }^{1} \mathrm{H}$ NMR $\left(400 \mathrm{MHz}, \mathrm{CDCl}_{3}\right) \delta 8.39(\mathrm{~s}, 1 \mathrm{H})$, $7.50(\mathrm{~d}, J=8.0 \mathrm{~Hz}, 1 \mathrm{H}), 7.46-7.33(\mathrm{~m}, 7 \mathrm{H}), 7.32-7.18(\mathrm{~m}, 5 \mathrm{H})$, 7.10-7.04 (m, 1H), 5.75 (s, 2H), 5.28 (s, 1H); ${ }^{13} \mathrm{C}$ NMR (101 MHz, $\left.\mathrm{CDCl}_{3}\right) \delta 175.0,139.4,137.2,136.3,132.2,129.2(2 \mathrm{C}), 129.0(2 \mathrm{C})$, 128.7 (2C), 128.6 (2C), 128.6, 127.5, 127.1, 122.8, 120.8, 120.6, 111.4, 110.2, 49.8; FT-IR ( $\mathrm{NaCl}, \mathrm{cm}^{-1}$ ): 3304, 2945, 2862, 1666, 1597, 1504, 1449, 1236, 1036; HRMS (TOF-ES): calcd for $\mathrm{C}_{22} \mathrm{H}_{18} \mathrm{~N}_{2} \mathrm{NaO}(\mathrm{M}+\mathrm{Na})^{+}: 349.1311$, found 349.1321 (2.9 ppm).

2-(4-Ethylphenyl)-2-(2-phenyl-1H-indol-3-yl)acetamide (7ab). Colorless solid, mp $142-145^{\circ} \mathrm{C}, R_{\mathrm{f}} 0.44$ (EtOAc/petroleum ether, $1: 1) ;{ }^{1} \mathrm{H}$ NMR $\left(400 \mathrm{MHz}, \mathrm{CDCl}_{3}\right) \delta 8.30(\mathrm{~s}, 1 \mathrm{H}), 7.55(\mathrm{~d}, J=8.0$ $\mathrm{Hz}, 1 \mathrm{H}), 7.51-7.34(\mathrm{~m}, 7 \mathrm{H}), 7.31-7.17$ (m, 2H), 7.10 (dd, $J=17.5$, $7.9 \mathrm{~Hz}, 3 \mathrm{H}), 5.68(\mathrm{~d}, J=61.1 \mathrm{~Hz}, 2 \mathrm{H}), 5.26(\mathrm{~s}, 1 \mathrm{H}), 2.61(\mathrm{q}, J=7.6$ $\mathrm{Hz}, 2 \mathrm{H}), 1.21$ (t, $J=7.6 \mathrm{~Hz}, 3 \mathrm{H}) ;{ }^{13} \mathrm{C} \mathrm{NMR}\left(101 \mathrm{MHz}, \mathrm{CDCl}_{3}\right)$ $\delta 175.1,143.0,137.1,136.6,136.3,132.3,129.1$ (2C), 128.9 (2C), 128.7 (2C), 128.6, 128.2 (2C), 127.7, 122.8, 120.9, 120.6, 111.3, 110.5, 49.4, 28.6, 15.5; FT-IR ( $\left.\mathrm{NaCl}, \mathrm{cm}^{-1}\right)$ : 3442, 3173, 2938, 1663, 1583, 1487, 1466, 1243; HRMS (TOF-ES): calcd for $\mathrm{C}_{24} \mathrm{H}_{22} \mathrm{~N}_{2} \mathrm{NaO}(\mathrm{M}+\mathrm{H})^{+}: 377.1624$, found 377.1628 (1.0 ppm).

2-(4-Methoxyphenyl)-2-(2-phenyl-1H-indol-3-yl)acetamide (7ac). Colorless solid, mp 102-104 ${ }^{\circ} \mathrm{C}, R_{\mathrm{f}} 0.79$ (EtOAc/petroleum ether $1: 1) ;{ }^{1} \mathrm{H} \mathrm{NMR}\left(400 \mathrm{MHz}, \mathrm{CDCl}_{3}\right) \delta 8.72(\mathrm{~s}, 1 \mathrm{H}), 7.62(\mathrm{~d}, J=7.3 \mathrm{~Hz}$, $2 \mathrm{H}), 7.54(\mathrm{~d}, J=8.1 \mathrm{~Hz}, 1 \mathrm{H}), 7.41(\mathrm{t}, J=7.6 \mathrm{~Hz}, 2 \mathrm{H}), 7.34-7.27(\mathrm{~m}$,
$2 \mathrm{H}), 7.21(\mathrm{~d}, J=8.8 \mathrm{~Hz}, 2 \mathrm{H}), 6.97(\mathrm{dd}, J=8.2,1.3 \mathrm{~Hz}, 1 \mathrm{H}), 6.88-$ $6.74(\mathrm{~m}, 3 \mathrm{H}), 5.75(\mathrm{~d}, J=60.5 \mathrm{~Hz}, 2 \mathrm{H}), 5.01(\mathrm{~s}, 1 \mathrm{H}), 3.76(\mathrm{~s}, 3 \mathrm{H}) ;{ }^{13} \mathrm{C}$ NMR (101 MHz, $\mathrm{CDCl}_{3}$ ) $\delta 175.8,158.8,138.8,137.3,133.7,132.4$, 132.0, 130.1 (2C), 129.1 (2C), 128.6, 127.9, 125.3 (2C), 121.5, 121.1, 114.3 (2C), 111.3, 99.7, 58.3, 55.4; FT-IR ( $\left.\mathrm{NaCl}, \mathrm{cm}^{-1}\right): 3331,2938$, 2862, 1669, 1577, 1487, 1456, 1088, 1005; HRMS (TOF-ES): calcd for $\mathrm{C}_{23} \mathrm{H}_{20} \mathrm{~N}_{2} \mathrm{NaO}_{2}(\mathrm{M}+\mathrm{Na})^{+}: 379.1417$, found 379.1409 (2.1 ppm).

2-(2-Fluorophenyl)-2-(2-phenyl-1H-indol-3-yl)acetamide (7ad). Colorless solid, mp 215-217 ${ }^{\circ} \mathrm{C}, R_{\mathrm{f}} 0.48$ (EtOAc/petroleum ether $1: 1) ;{ }^{1} \mathrm{H}$ NMR (400 MHz, DMSO- $\left.d_{6}\right) \delta 11.43(\mathrm{~s}, 1 \mathrm{H}), 7.63(\mathrm{~d}, J=$ $8.1 \mathrm{~Hz}, 1 \mathrm{H}), 7.52-7.34(\mathrm{~m}, 6 \mathrm{H}), 7.24$ (s, 2H), 7.12 (dd, $J=16.3$, 8.4 $\mathrm{Hz}, 3 \mathrm{H}), 7.04(\mathrm{t}, J=7.1 \mathrm{~Hz}, 2 \mathrm{H}), 6.95(\mathrm{t}, J=7.4 \mathrm{~Hz}, 1 \mathrm{H}), 5.31(\mathrm{~s}$, $1 \mathrm{H}) ;{ }^{13} \mathrm{C}$ NMR (101 MHz, DMSO- $\left.d_{6}\right) \delta 172.4,160.1(\mathrm{~d}, J=244.3$ $\mathrm{Hz}), 136.4,136.1,132.5,130.2$ (d, $J=3.8 \mathrm{~Hz}), 128.6$ (2C), 128.4 (d, $J=8.0 \mathrm{~Hz}), 128.3(2 \mathrm{C}), 128.2,127.84,127.75,123.9(\mathrm{~d}, J=3.0 \mathrm{~Hz})$, 121.4, 120.4, 119.0, $114.8(\mathrm{~d}, J=21.7 \mathrm{~Hz}), 111.3,107.6,42.8(\mathrm{~d}, J=$ $1.1 \mathrm{~Hz}$ ); FT-IR ( $\mathrm{NaCl}, \mathrm{cm}^{-1}$ ): 3442, 3180, 3049, 1663, 1583, 1487, 1452, 1398, 1232; HRMS (TOF-ES): calcd for $\mathrm{C}_{22} \mathrm{H}_{17} \mathrm{FN}_{2} \mathrm{NaO}(\mathrm{M}+$ $\mathrm{Na})^{+}: 367.1217$, found $367.1213(1.1 \mathrm{ppm})$.

2-(4-Chlorophenyl)-2-(2-phenyl-1H-indol-3-yl)acetamide (7ae). Colorless solid, mp $138-141{ }^{\circ} \mathrm{C}, R_{\mathrm{f}} 0.45$ (EtOAc/petroleum ether 1 : 1); ${ }^{1} \mathrm{H}$ NMR $\left(400 \mathrm{MHz}, \mathrm{CDCl}_{3}\right) \delta 8.41(\mathrm{~s}, 1 \mathrm{H}), 7.48-7.33(\mathrm{~m}, 7 \mathrm{H})$, $7.28-7.19(\mathrm{~m}, 5 \mathrm{H}), 7.08$ (t, $J=7.5 \mathrm{~Hz}, 1 \mathrm{H}), 5.69$ (d, $J=26.4 \mathrm{~Hz}$, $2 \mathrm{H}), 5.23(\mathrm{~s}, 1 \mathrm{H}) ;{ }^{13} \mathrm{C} \mathrm{NMR}\left(101 \mathrm{MHz}, \mathrm{CDCl}_{3}\right) \delta 174.4,137.8,137.4$, 136.3, 132.9, 132.0, 130.4 (2C), 129.3 (2C), 128.8, 128.7 (2C), 128.6 (2C), 127.3, 123.0, 120.8, 120.7, 111.4, 109.6, 48.9; FT-IR (NaCl, $\mathrm{cm}^{-1}$ ): 3435, 3166, 1652, 1590, 1490, 1459, 1404; HRMS (TOF-ES): calcd for $\mathrm{C}_{22} \mathrm{H}_{17} \mathrm{ClN}_{2} \mathrm{NaO}(\mathrm{M}+\mathrm{Na})^{+}: 383.0922$, found 383.0927 (1.3 ppm).

2-(4-Ethylphenyl)-2-(2-methyl-1H-indol-3-yl)acetamide (7bb). Light brown solid, $\mathrm{mp} 72-75{ }^{\circ} \mathrm{C}, R_{\mathrm{f}} 0.26$ (EtOAc/petroleum ether $1: 1) ;{ }^{1} \mathrm{H}$ NMR (400 MHz, $\left.\mathrm{CDCl}_{3}\right) \delta 8.05(\mathrm{~s}, 1 \mathrm{H}), 7.42-7.35(\mathrm{~m}$, $1 \mathrm{H})$, 7.29-7.23 (m, 3H), 7.13-7.06 (m, 3H), 7.06-6.98 (m, 1H), $5.74(\mathrm{~d}, J=37.0 \mathrm{~Hz}, 2 \mathrm{H}), 5.13(\mathrm{~s}, 1 \mathrm{H}), 2.60(\mathrm{q}, J=7.6 \mathrm{~Hz}, 2 \mathrm{H})$, $2.34(\mathrm{~s}, 3 \mathrm{H}), 1.20(\mathrm{t}, J=7.6 \mathrm{~Hz}, 3 \mathrm{H}) ;{ }^{13} \mathrm{C} \mathrm{NMR}\left(101 \mathrm{MHz}, \mathrm{CDCl}_{3}\right)$ $\delta 175.5,143.0,136.5,133.2,128.8,128.2,127.6,121.5,119.9$, 119.1, 110.6, 109.7, 49.3, 28.6, 15.6, 12.3; FT-IR ( $\left.\mathrm{NaCl}, \mathrm{cm}^{-1}\right)$ : 3469, 3304, 2986, 1656, 1597, 1456, 1373, 1246; HRMS (TOF-ES): calcd for $\mathrm{C}_{19} \mathrm{H}_{20} \mathrm{~N}_{2} \mathrm{NaO}(\mathrm{M}+\mathrm{Na})^{+}: 315.1468$, found 315.1465 (1.0 ppm).

2-(4-Chlorophenyl)-2-(2-methyl-1H-indol-3-yl)acetamide (7be). Light brown solid, mp 101-103 ${ }^{\circ} \mathrm{C}, R_{\mathrm{f}} 0.14$ (EtOAc/petroleum ether $1: 1$ ); ${ }^{1} \mathrm{H}$ NMR (400 MHz, $\mathrm{CDCl}_{3}$ ) $\delta 8.13$ (s, 1H), 7.34-7.28 (m, 2H), 7.28-7.23 (m, 4H), 7.16-7.09 (m, 1H), 7.07-7.00 (m, 1H), $5.80(\mathrm{~d}, J=8.5 \mathrm{~Hz}, 2 \mathrm{H}), 5.11(\mathrm{~s}, 1 \mathrm{H}), 2.34(\mathrm{~s}, 3 \mathrm{H}) ;{ }^{13} \mathrm{C} \mathrm{NMR}(101$ $\mathrm{MHz}, \mathrm{CDCl}_{3}$ ) $\delta 174.6,137.6,135.5,133.4,132.9,130.3$ (2C), 128.7 (2C), 127.3, 121.9, 120.3, 118.9, 110.8, 109.1, 48.8, 12.3; FT-IR $\left(\mathrm{NaCl}, \mathrm{cm}^{-1}\right): 3311,2924,1666,1604,1504,1456,1246,1177$, 1026; HRMS (TOF-ES): calcd for $\mathrm{C}_{17} \mathrm{H}_{15} \mathrm{ClN}_{2} \mathrm{NaO}(\mathrm{M}+\mathrm{Na})^{+}$: 321.0765 , found 321.0758 (2.2 ppm).

2-(2-Methyl-1H-indol-3-yl)acetamide (7bf). ${ }^{13}$ Colorless crystals, mp 126-127 ${ }^{\circ} \mathrm{C}$; physical and spectral properties of this compound were identical to those of commercially available authentic sample.

2-Phenyl-2-(2-( $\boldsymbol{p}$-tolyl)-1H-indol-3-yl)acetamide (7ca). Light brown solid, mp 143-145 ${ }^{\circ} \mathrm{C}, R_{\mathrm{f}} 0.49$ (EtOAc/petroleum ether 1 : 1); ${ }^{1} \mathrm{H}$ NMR (400 MHz, $\mathrm{CDCl}_{3}$ ) $\delta 8.30$ (s, 1H), 7.47 (dd, $J=$ 
12.7, $4.7 \mathrm{~Hz}, 2 \mathrm{H}), 7.43-7.32(\mathrm{~m}, 5 \mathrm{H}), 7.32-7.15(\mathrm{~m}, 5 \mathrm{H}), 7.07(\mathrm{t}, J$ $=7.5 \mathrm{~Hz}, 1 \mathrm{H}), 5.72(\mathrm{~d}, J=35.3 \mathrm{~Hz}, 2 \mathrm{H}), 5.28(\mathrm{~s}, 1 \mathrm{H}), 2.40(\mathrm{~s}, 3 \mathrm{H})$; ${ }^{13} \mathrm{C}$ NMR $\left(101 \mathrm{MHz}, \mathrm{CDCl}_{3}\right) \delta 175.1,139.5,138.7,137.4,136.2$, 129.9 (2C), 129.3 (2C), 129.0 (2C), 128.6 (2C), 128.5, 127.6, 127.0, 122.6, 120.7, 120.5, 111.3, 109.9, 49.8, 21.4; FT-IR ( $\left.\mathrm{NaCl}, \mathrm{cm}^{-1}\right)$ : 3442, 3173, 2917, 1666, 1587, 1494, 1446, 1391; HRMS (TOF-ES): calcd for $\mathrm{C}_{23} \mathrm{H}_{20} \mathrm{~N}_{2} \mathrm{NaO}(\mathrm{M}+\mathrm{Na})^{+}: 363.1468$, found 363.1458 (2.7 ppm).

2-(2-(Naphthalen-2-yl)-1H-indol-3-yl)-2-phenylacetamide (7da) ${ }^{6}$ Colorless solid, mp 332-333 ${ }^{\circ} \mathrm{C}, R_{\mathrm{f}} 0.57$ (EtOAc/petroleum ether $1: 1) ;{ }^{1} \mathrm{H}$ NMR (400 MHz, $\left.\mathrm{CDCl}_{3}\right) \delta 8.40(\mathrm{~s}, 1 \mathrm{H}), 7.97(\mathrm{~s}, 1 \mathrm{H}), 7.90$ $(\mathrm{d}, J=8.5 \mathrm{~Hz}, 1 \mathrm{H}), 7.87-7.79(\mathrm{~m}, 2 \mathrm{H}), 7.64(\mathrm{dd}, J=8.5,1.6 \mathrm{~Hz}, 1 \mathrm{H})$, $7.56-7.47(\mathrm{~m}, 3 \mathrm{H}), 7.44(\mathrm{~d}, J=8.1 \mathrm{~Hz}, 1 \mathrm{H}), 7.36(\mathrm{~d}, J=7.3 \mathrm{~Hz}, 2 \mathrm{H})$, $7.32-7.20(\mathrm{~m}, 4 \mathrm{H}), 7.07(\mathrm{t}, J=7.5 \mathrm{~Hz}, 1 \mathrm{H}), 5.34(\mathrm{~s}, 1 \mathrm{H}), 4.70(\mathrm{~s}, 2 \mathrm{H})$; ${ }^{13} \mathrm{C}$ NMR $\left(101 \mathrm{MHz}, \mathrm{CDCl}_{3}\right) \delta 155.8,140.1,137.2,136.4,133.5$, 133.0, 129.6, 128.9 (3C), 128.5 (2C), 128.4, 128.3, 128.1, 127.9, 126.9, 126.8, 126.8, 126.1, 122.8, 121.0, 120.7, 111.3, 110.9, 45.0; FT-IR ( $\mathrm{NaCl}, \mathrm{cm}^{-1}$ ): 3393, 3049, 1645, 1570, 1439, 1329, 1005; HRMS (TOF-ES): calcd for $\mathrm{C}_{26} \mathrm{H}_{20} \mathrm{~N}_{2} \mathrm{NaO}(\mathrm{M}+\mathrm{Na})^{+}$: 399.1468, found $399.1476(2.0 \mathrm{ppm})$.

2-(1H-Indol-3-yl)acetamide (7ef). ${ }^{\mathbf{1 4}}$ Colorless crystals, $\mathrm{mp}$ 150-151 ${ }^{\circ} \mathrm{C}$; physical and spectral properties of this compound were identical to those of commercially available authentic sample.

2-(4-Methoxyphenyl)-2-phenylacetamide (9aa). Yellowish oil, $R_{\mathrm{f}} 0.33$ (EtOAc/petroleum ether $\left.1: 1\right) ;{ }^{1} \mathrm{H} \mathrm{NMR}\left(400 \mathrm{MHz}, \mathrm{CDCl}_{3}\right)$ $\delta 7.34-7.22(\mathrm{~m}, 5 \mathrm{H}), 7.17(\mathrm{~d}, J=8.6 \mathrm{~Hz}, 2 \mathrm{H}), 6.85(\mathrm{~d}, J=8.7 \mathrm{~Hz}$, 2H), 4.89 (s, 1H), 4.56 (s, 2H), 3.79 (s, 3H); ${ }^{13} \mathrm{C}$ NMR (101 MHz, $\left.\mathrm{CDCl}_{3}\right) \delta 158.8,154.7,140.0,131.7,130.1$ (2C), 129.0 (2C), 128.7 (2C), 127.2 (2C), 114.1, 55.4, 52.7; FT-IR ( $\left.\mathrm{NaCl}, \mathrm{cm}^{-1}\right)$ : 3380, 2993, 2883, 1656, 1604, 1580, 1511, 1456, 1253, 1181, 1033; HRMS (TOF-ES): calcd for $\mathrm{C}_{15} \mathrm{H}_{15} \mathrm{NNaO}_{2}(\mathrm{M}+\mathrm{Na})^{+}$264.0995, found, 264.0998 (1.1 ppm).

2-(4-Methoxyphenyl)acetamide (9af).${ }^{15}$ Colorless crystals, $\mathrm{mp}$ 188-189 ${ }^{\circ} \mathrm{C}$; physical and spectral properties of this compound were identical to those of commercially available authentic sample.

2-(4-Methoxyphenyl)propanamide (9ag). ${ }^{16}$ Colorless crystals, mp 109-110 ${ }^{\circ} \mathrm{C}$; physical and spectral properties of this compound were identical to those of commercially available authentic sample.

2-(2,3-Dihydrobenzo[b][1,4] dioxin-6-yl)-2-phenylacetamide (9ba). Light brown solid, mp $74-76{ }^{\circ} \mathrm{C}, R_{\mathrm{f}} 0.65$ (EtOAc/petroleum ether, $1: 1) ;{ }^{1} \mathrm{H}$ NMR (400 MHz, $\mathrm{CDCl}_{3}$ ) $\delta$ 7.34-7.27 (m, 2H), 7.27-7.20 (m, $3 \mathrm{H}), 6.80(\mathrm{~d}, J=8.3 \mathrm{~Hz}, 1 \mathrm{H}), 6.76(\mathrm{~d}, J=2.0 \mathrm{~Hz}, 1 \mathrm{H}), 6.71$ (dd, $J=$ 8.3, $2.1 \mathrm{~Hz}, 1 \mathrm{H}), 6.00$ (d, J=273.5 Hz, 2H), 4.80 (s, 1H), 4.19 (s, 4H); ${ }^{13} \mathrm{C}$ NMR $\left(101 \mathrm{MHz}, \mathrm{CDCl}_{3}\right) \delta 175.0,143.7,142.9,139.4,132.6,128.9$ (2C), 128.8 (2C), 127.3, 121.9, 117.8, 117.6, 64.4 (2C), 58.1; FT-IR $\left(\mathrm{NaCl}, \mathrm{cm}^{-1}\right)$ : 3428, 3193, 1659, 1590, 1497, 1284, 1256, 1070; HRMS (TOF-ES): calcd for $\mathrm{C}_{16} \mathrm{H}_{15} \mathrm{NNaO}_{3}(\mathrm{M}+\mathrm{Na})^{+}: 392.0944$, found 392.0942 (0.5 ppm).

$2-(2,3,5,6,8,9,11,12-O c t a h y d r o b e n z o[b][1,4,7,10,13]$ pentaoxacyclopentadecin-15-yl)-2-phenylacetamide (9ca). Yellowish oil, $R_{\mathrm{f}} 0.43$ (EtOH); ${ }^{1} \mathrm{H}$ NMR $\left(400 \mathrm{MHz}, \mathrm{CDCl}_{3}\right) \delta 7.41-7.19(\mathrm{~m}, 5 \mathrm{H})$, 6.83-6.70 (m, 13H), $4.86(\mathrm{~s}, 1 \mathrm{H}), 4.60(\mathrm{~s}, 2 \mathrm{H}), 4.19-4.01(\mathrm{~m}, 4 \mathrm{H})$, 3.94-3.81 (m, 4H), $3.74(\mathrm{~s}, 8 \mathrm{H}) ;{ }^{13} \mathrm{C} \mathrm{NMR} \mathrm{(101} \mathrm{MHz,} \mathrm{CDCl}_{3}$ ) $\delta$ 154.7, 149.0, 148.1, 139.9, 132.7, 129.1 (2C), 128.7 (2C), 127.2,
121.9, 114.9, 113.7, 72.0 (2C), 70.4 (2C), 69.6 (2C), 68.9, 68.8, 53.1; FT-IR ( $\mathrm{NaCl}, \mathrm{cm}^{-1}$ ): 3359, 2945, 2883, 1663, 1608, 1551, 1267, 1132, 1053; HRMS (TOF-ES): calcd for $\mathrm{C}_{22} \mathrm{H}_{27} \mathrm{NNaO}_{6}(\mathrm{M}+$ $\mathrm{Na})^{+}$: 424.1731, found $424.1727(0.9 \mathrm{ppm})$.

\section{Acknowledgements}

Financial support for this work was provided by Russian Science Foundation (grant \#14-13-01108).

\section{Notes and references}

1 For isolation and biological studies, see: (a) M. E. Adams, R. L. Carney, F. E. Enderlin, E. T. Fu, M. A. Jarema, J. P. Li, C. A. Miller, D. A. Schooley, M. J. Shapiro and V. J. Venema, Biochem. Biophys. Res. Commun., 1987, 148, 678; (b) M. S. Davies, M. P. Baganoff, E. V. Grishin, T. H. Lanthorn, T. M. Volkova, G. B. Watson and R. C. Wiegand, Eur. J. Pharmacol., 1992, 227, 51; (c) W. S. Skinner, M. E. Adams, G. B. Quistad, H. Kataoka, B. J. Cesarin, F. E. Enderlin and D. A. Schooley, J. Biol. Chem., 1989, 264, 2150. For total synthesis, see: (d) V. J. Jasys, P. R. Kelbaugh, D. M. Nason, D. Phillips, N. A. Saccomano and R. A. Volkmann, Tetrahedron Lett., 1988, 29, 6223.

2 (a) H. E. Colley, M. Muthana, S. J. Danson, L. V. Jackson, M. L. Brett, J. Harrison, S. F. Coole, D. P. Mason, L. R. Jennings, M. Wong, V. Tulasi, D. Norman, P. M. Lockey, L. Williams, A. G. Dossetter, E. J. Griffen and M. J. Thompson, J. Med. Chem., 2015, 58, 9309; (b) A. Wienecke and G. Bacher, Cancer Res., 2009, 69, 171; (c) P.-F. Yu, H. Chen, J. Wang, C.-X. He, B. Cao, M. Li, N. Yang, Z.-Y. Lei and M.-S. Cheng, Chem. Pharm. Bull., 2008, 56, 831; (d) G. Bacher, B. Nickel, P. Emig, U. Vanhoefer, S. Seeber, A. Shandra, T. Klenner and T. Beckers, Cancer Res., 2001, 61, 392.

3 (a) E. Salgado, J. R. Maneiro, L. Carmona and J. J. GomezReino, Ann. Rheum. Dis., 2014, 73, 871; (b) C. Dominguez, D. A. Powers and N. Tamayo, Curr. Opin. Drug Discovery Dev., 2005, 8, 421; (c) D. M. Goldstein, A. Kuglstatter, Y. Lou and M. J. Soth, J. Med. Chem., 2010, 53, 2345; (d) S. N. Nikas and A. A. Drosos, Curr. Opin. Invest. Drugs, 2004, 5, 1205.

4 See, for example: (a) H. R. Schumacher, J. A. Boice, D. I. Daikh, S. Mukhopadhyay, K. Malmstrom, J. Ng, G. A. Tate and J. Molina, BMJ, 2002, 324, 1488; (b) M. Gotteland, S. Cruchet and S. Verbeke, Aliment. Pharmacol. Ther., 2001, 15, 11; (c) I. Jurna and K. Brune, J. Pain, 1990, 41, 71; (d) I. Jurna, B. Spohrer and R. Bock, J. Pain, 1992, 49, 249; (e) K. D. Rainsford, P. I. Stetsko, S. P. Sirko and S. Debski, J. Pharm. Pharmacol., 2003, 55, 661. 5 (a) A. V. Aksenov, A. N. Smirnov, N. A. Aksenov, I. V. Aksenova, L. V. Frolova, A. Kornienko, I. V. Magedov and M. Rubin, Chem. Commun., 2013, 49, 9305; (b) A. V. Aksenov, A. N. Smirnov, N. A. Aksenov, I. V. Aksenova, A. S. Bijieva and M. Rubin, Org. Biomol. Chem., 2014, 12, 9786; (c) A. V. Aksenov, A. N. Smirnov, N. A. Aksenov, 
I. V. Aksenova, J. P. Matheny and M. Rubin, $R S C A d v ., 2015,5$, 8647.

6 A. V. Aksenov, A. N. Smirnov, I. V. Magedov, M. R. Reisenauer, N. A. Aksenov, I. V. Aksenova, A. L. Pendleton, G. Nguyen, R. K. Johnston, M. Rubin, A. De Carvalho, R. Kiss, V. Mathieu, F. Lefranc, J. Correa, D. A. Cavazos, A. J. Brenner, B. A. Bryan, S. Rogelj, A. Kornienko and L. Frolova, J. Med. Chem., 2015, 58, 2206.

7 A. V. Aksenov, N. A. Aksenov, Z. V. Dzhandigova, I. V. Aksenova, L. G. Voskressensky, A. N. Smirnov and M. Rubin, Chem. Heterocycl. Compd., 2016, 52, 299.

8 A. V. Aksenov, N. A. Aksenov, Z. V. Dzhandigova, D. A. Aksenov and M. Rubin, RSC Adv., 2015, 5, 106492.

9 (a) R. M. Gipson, F. H. Pettit, C. G. Skinner and W. Shive, J. Org. Chem., 1963, 28, 1425; (b) K. Ahlford and H. Adolfsson, Catal. Commun., 2011, 12, 1118; (c) H. W. Thompson and S. Y. Rashid, J. Org. Chem., 2002, 67, 2813.

10 (a) H. Fukuzawa, Y. Ura and Y. Kataoka, J. Organomet. Chem., 2011, 696, 3643; (b) P. G. Mattingly and M. J. Miller, J. Org.
Chem., 1980, 45, 410; (c) S. Rakshit, C. Grohmann, T. Besset and F. Glorius, J. Am. Chem. Soc., 2011, 133, 2350; (d) F. Yang and L. Ackermann, J. Org. Chem., 2014, 79, 12070; (e) C. Grohmann, H. Wang and F. Glorius, Org. Lett., 2012, 14, 656; ( $f$ ) E. Wagner, L. Becan and E. Nowakowska, Bioorg. Med. Chem., 2004, 12, 265.

11 K. Sugihara and K. Tatsumi, Arch. Biochem. Biophys., 1986, 247, 289.

12 G. P. Dhareshwar, P. N. Chhaya and B. D. Hosangadi, Indian J. Chem., Sect. B: Org. Chem. Incl. Med. Chem., 1980, 19, 831.

13 H. J. Teuber, K. Schumann, U. Reinehr and A. Gholami, Liebigs Ann. Chem., 1983, 1744.

14 C. S. Yi, T. N. Zeczycki and S. V. Lindeman, Organometallics, 2008, 27, 2030.

15 A. R. Katritzky, B. Pilarski and L. Urogdi, Synthesis, 1989, 945. 16 T. D. Booth, W. J. Lough, M. Saeed, T. A. G. Noctor and I. W. Wainer, Chirality, 1997, 9, 173. 